\title{
Determination of Correction Values of Operating Reliability of Assembly Components of the Assembly-Front Spinner Spinning Box (R1 Rieter) on the Basis of Operating Data
}

\author{
Slobodan Stefanović1, Korabayev Sherzod Ahmadjonovich ${ }^{2 *}$, \\ Shoxobiddinova Dilafruz Erkinzonqizi ${ }^{2}$ \\ ${ }^{1}$ Academy of Technical and Educational Vocational Studies, Nis, Serbia \\ ${ }^{2}$ Department of Technology of Products of Textile Industry, Namangan Institute of Engineering and Technology, Namangan, \\ Uzbekistan \\ Email: sherzod.korabayev@gmail.com
}

How to cite this paper: Stefanović, S., Ahmadjonovich, K.S. and Erkinzonqizi, S.D. (2021) Determination of Correction Values of Operating Reliability of Assembly Components of the Assembly-Front Spinner Spinning Box (R1 Rieter) on the Basis of Operating Data. Engineering, 13, 565-573. https://doi.org/10.4236/eng.2021.1311041

Received: July 1, 2021

Accepted: November 8, 2021

Published: November 11, 2021

Copyright $\odot 2021$ by author(s) and Scientific Research Publishing Inc. This work is licensed under the Creative Commons Attribution International License (CC BY 4.0).

http://creativecommons.org/licenses/by/4.0/

\begin{abstract}
The optimal safety model includes the values of the operation of the components with the allowed risk, and it is clearly seen that the belt of absolutely safe operation of the analyzed circuit is located between the values of the displayed dependence curves $M_{\xi}(t)_{B P}=f(t)$. AND also, analyses have been carried out to ensure the flawless operation of the OE machine and the consistency of product quality.
\end{abstract}

\section{Keywords}

Surrender Box, Open-End (OE), Reliability, Exploitation, Spinning, Yarn, Tension

\section{Introduction-Description of the Box Spinning Assembly (Heart OE Spinner)}

The power transition system of the spinning box assembly is shown in Figure 1 and consists of the following components which are classified on the basis of the processing flow of the carded sliver (raw material to be processed).

When the operational values of reliability are determined, which express the approximate values of reliability of the components of the analyzed assembly with maximum safety (areas of their safe operation time and areas of reduction 


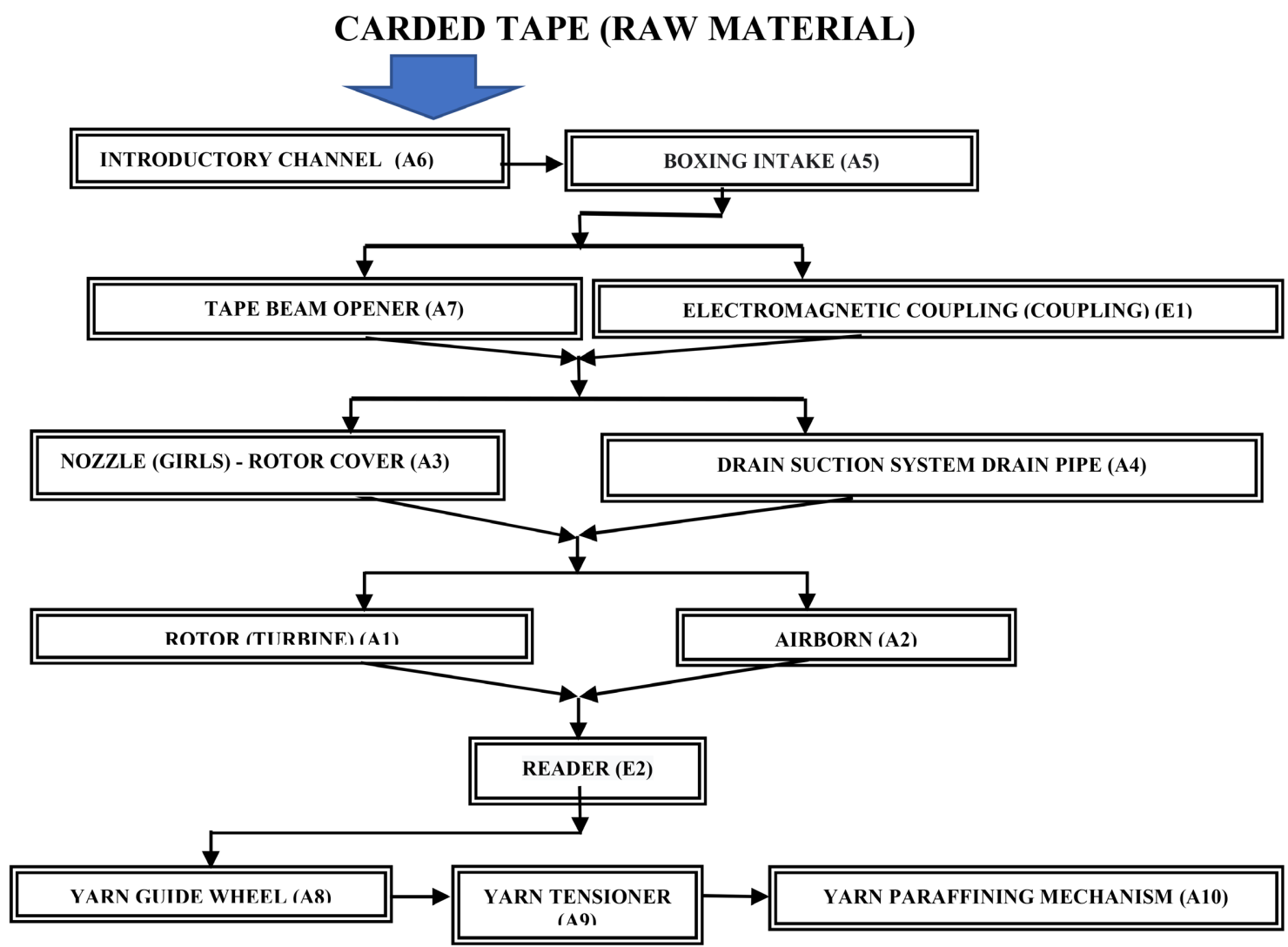

Figure 1. Assembly power transmission system—spinning box.

of their reliability), their correction values were used for more precise determination. This was aimed at obtaining the most accurate reliability values for determining the total transfer function of the reliability of the components of the analyzed assembly [1] [2] [3].

The correction values of reliability from the exploitation data are shown in Figure 2 to Figure 3, and their tabular values within the presented figures.

Reliability correction values are obtained as a quotient of the empirical distribution density function from empirical values $\left(f_{e}(t)\right)$ and failure intensity functions $\left(\lambda_{e}(t)\right)$ for the time interval of the operational operation of the components of the assemblies (the analysis included the service life of the components of the assembly in the duration $13000 \leq \Delta t_{i} \leq 21000$ hours) and are determined by the expression:

$$
P_{i}(t)=\frac{f_{e_{i}}(t)}{\lambda_{e_{i}}(t)} .
$$

The obtained correction values will further serve in the formation of tables of values of the transfer functions of the spinning box assembly $G_{B P}(t)$ on the basis of which the shapes of the curves are determined $f\left(G_{B P}(t), t\right)$ which determine the form of statistical distribution of reliability, i.e. the shape of the curve adopts the distribution of reliability that best suits their shape [4] [5].

Conclusion: From the shape of the curve of the correction values of the reliability 


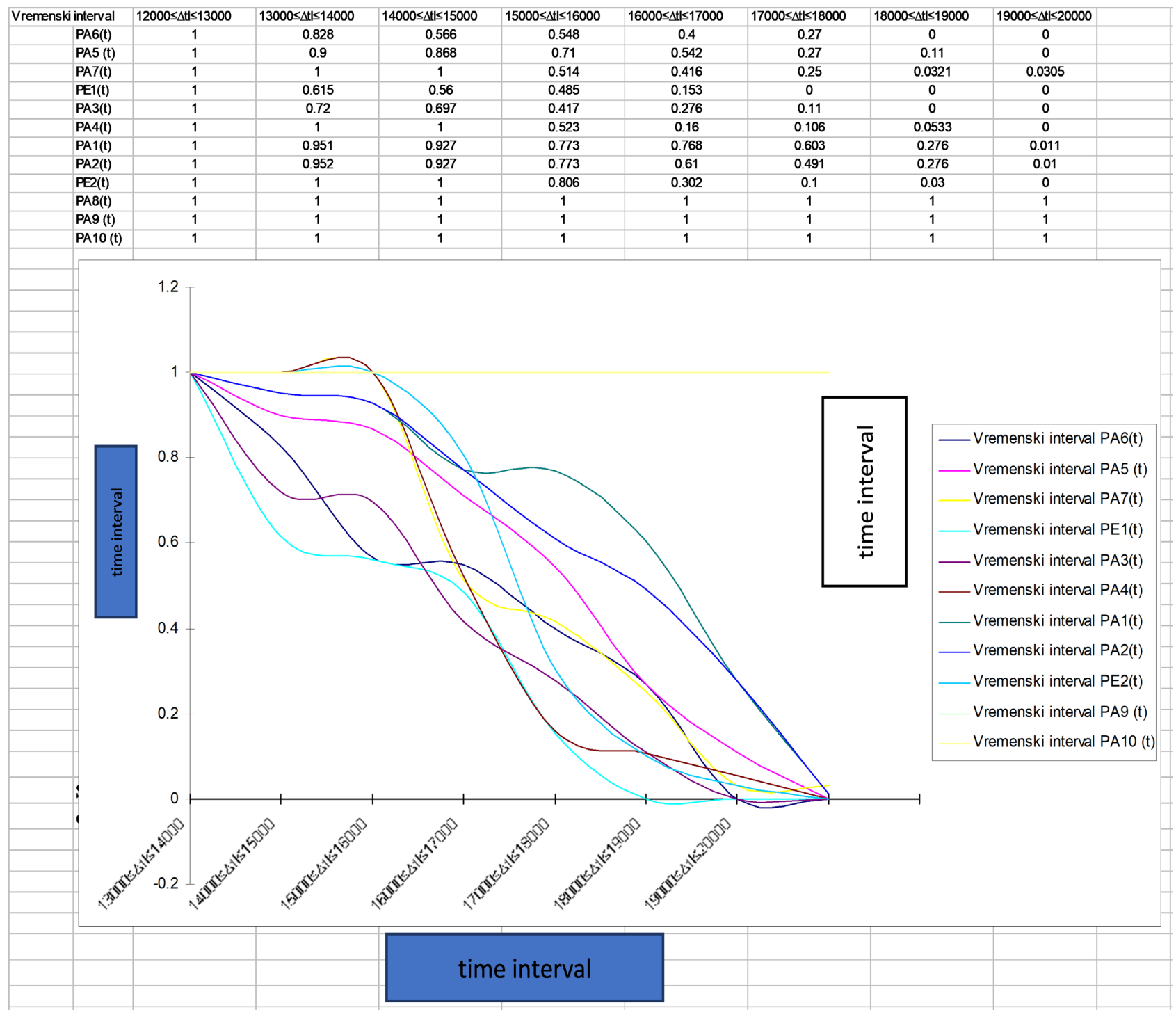

Figure 2. Diagram of correction values of the reliability of the components of the analyzed spinning box assemblies on the basis of empirical data on which the procedures of preventive maintenance technology have not been applied.

of the components of the analyzed assembly $P_{i}(t)$, i.e. according to the slope of the curve, confidence intervals can be analytically predicted which will later be used as a basis in determining the relevant reliability (reliability obtained from the statistical distribution) [6] [7] [8].

The analysis showed the following conclusions:

1) Analysis of the reliability of the operation of the components of the analyzed assemblies without the application of preventive maintenance technology procedures:

- Components A8, A9, A10 have the highest reliability in operation, whose reliability is maximum and amounts $P_{A 8}(t)=P_{A 9}(t)=P_{A 10}(t)=1.0$ and lasts in a time interval over $\Delta t_{i} \geq 20000(\mathrm{~h})$.

- Based on the shape of the curve $f\left(P_{i}(t), t\right)$, i.e. according to their slope, Table 1 shows the order of reliability values and component operating times. 


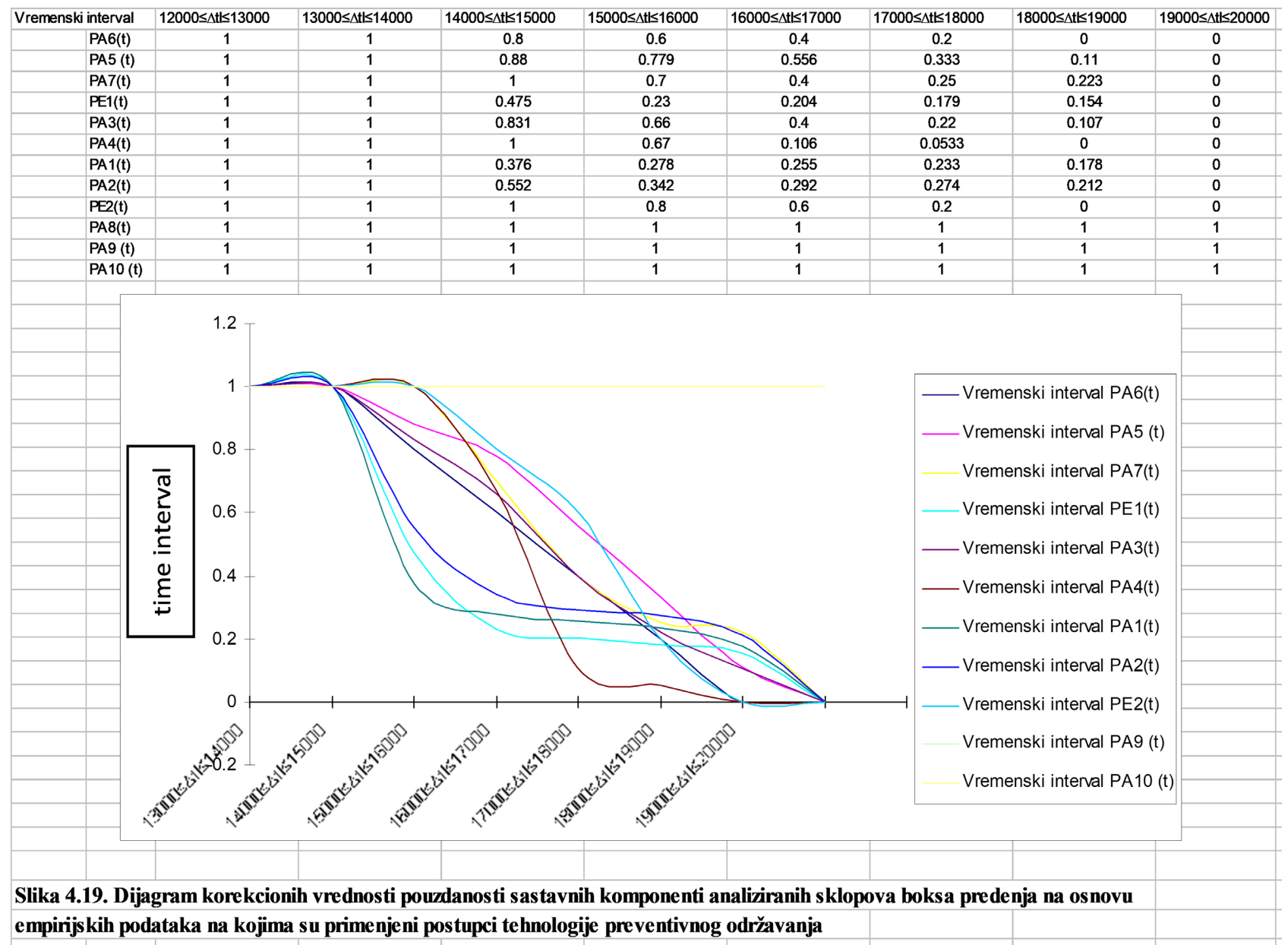

Figure 3. Diagram of correction values of the reliability of the components of the analyzed spinning box assemblies based on empirical data on which the procedures of preventive maintenance technology were applied.

Table 1. Values of the correction reliability interval depending on the analyzed time interval of operation of the components of the analyzed assemblies on which the procedures of preventive maintenance technology have not been applied.

Time interval of the analyzed Confidence interval for the anaComponent designation work of the component lyzed time interval

$\Delta t_{1} \leq \Delta t_{i} \leq \Delta t_{2} \quad \Delta P_{i 1} \leq \Delta P_{i} \leq \Delta P_{i 2}$

Boxing spinning assembly

$\begin{array}{lcc}\text { A6 } & 13,000 \div 14,000 & 1.0 \div 0.828 \\ \text { A5 } & 13,000 \div 14,000 & 1.0 \div 0.9 \div 0.868 \\ \text { A7 } & 13,000 \div 16,000 & 1.0 \div 0.514 \\ \text { E1 } & 13,000 \div 14,000 & 1.0 \div 0.615 \\ \text { A3 } & 13,000 \div 14,000 & 1.0 \div 0.72 \\ \text { A4 } & 13,000 \div 16,000 & 1.0 \div 0.523 \\ \text { A1 } & 13,000 \div 14,000 & 1.0 \div 0.951 \div 0.927 \\ \text { A2 } & 13,000 \div 14,000 & 1.0 \div 0.952 \div 0.927 \\ \text { E2 } & 13,000 \div 16,000 & 1.0 \div 0.806\end{array}$


The analysis included confidence intervals after the first lower value than the maximum.

2) Analysis of the reliability of the components of the analyzed assemblies with the application of preventive maintenance technology procedures:

- Components A8, A9, A10 have the highest reliability in operation, whose reliability is maximum and amounts $P_{A 8-0}(t)=P_{A 9-0}(t)=P_{A 10-0}(t)=1.0$ and lasts in a time interval over $\Delta t_{i} \geq 20000(\mathrm{~h})$.

- And lasts in a time interval over $f\left(P_{i-0}(t), t\right)$, i.e. according to their slope, the order of values of reliability and operating time of components during these reliability is shown in the table (Table 2). The analysis included confidence intervals after the first lower value than the maximum.

\section{Determination of the Statistical Method of Reliability Distribution of the Analyzed Assembly}

In order to determine the statistical method of reliability distribution, it is necessary to form models and determine the transfer reliability functions of the analyzed assembly [9] [10]. To determine the transfer function tables of the spinning box assembly $G_{B P}(t)$ reliability corrections were used $P_{i}(t)$

\subsection{Model Formation and Determination of Transfer Functions of Reliability of Analyzed Assembly Based on Empirical Data}

The formation of the model included the arrangement of the components of the assemblies according to the processing of the yarn, i.e. according to the labels in the order of the components in the failure tree.

The components are arranged in sequence on the assembly, from the introductory

Table 2. Values of the correction reliability interval depending on the analyzed time interval of the components of the analyzed assemblies on which the procedures of preventive maintenance technology have been applied.

\begin{tabular}{ccc}
\hline $\begin{array}{c}\text { Component } \\
\text { designation }\end{array}$ & $\begin{array}{c}\text { Time interval of the analyzed } \\
\text { work of the component } \\
\Delta t_{1-0} \leq \Delta t_{i-0} \leq \Delta t_{2-0}\end{array}$ & $\begin{array}{c}\text { Confidence interval for the } \\
\text { analyzed time interval } \\
\Delta P_{i-01} \leq \Delta P_{i-0} \leq \Delta P_{i-02}\end{array}$ \\
\hline Boxing spinning assembly & \\
A6 & $13,000 \div 15,000$ & $1.0 \div 0.8$ \\
A5 & $13,000 \div 15,000$ & $1.0 \div 0.88$ \\
A7 & $13,000 \div 16,000$ & $1.0 \div 0.7$ \\
E1 & $13,000 \div 15,000$ & $1.0 \div 0.475$ \\
A3 & $13,000 \div 15,000$ & $1.0 \div 0.831$ \\
A4 & $13,000 \div 16,300$ & $1.0 \div 0.67$ \\
A1 & $13,000 \div 15,000$ & $1.0 \div 0.376$ \\
A2 & $13,000 \div 15,000$ & $1.0 \div 0.522$ \\
E2 & $13,000 \div 16,300$ & $1.0 \div 0.8$ \\
\hline
\end{tabular}


channel to the yarn waxing mechanism in the spinning box.

For these reasons, the block diagram model is shown. The model is more complex and includes the arrangement of components in the spinning box, taking into account their functionality and purpose, so that the reduction of complex block diagram structures has been performed.

Based on the obtained final expressions of the transfer functions of the analyzed circuits $G_{P}(t)_{B P}$ for boxing spinning), and in them by replacing the reliability values of the components $P_{i}(t)$ for time intervals

$13000(\mathrm{~h}) \leq \Delta t_{i} \leq 20000(\mathrm{~h})$ tabular values are obtained by reliability significance belts, from which the reliability curves of the transfer functions of the analyzed assembly are constructed (Table 3 ).

1) Block diagram model of the transmission reliability function in a spinning box assembly

For solving reduction of this model, its step-by-step solution will be performed when obtaining the transfer function of the circuit reliability $G_{P}(t)_{B P}$. As can be seen from Figure 4, this is an open system of automatic reliability management [11] [12]. The model is shown in Figure 4.

\section{Step I: Determining partial reliability blocks}

$$
\begin{aligned}
& P_{p 1}(t)=P_{E 1}(t) \cdot P_{A 7}(t), P_{p 2}(t)=P_{A 4}(t)+P_{A 3}(t), \\
& P_{p 3}(t)=P_{A 1}(t) \cdot P_{A 2}(t), P_{p 4}(t)=P_{A 8}(t)+P_{E 2}(t), \\
& P_{P 5}(t)=P_{A 9}(t)+P_{A 10}(t)
\end{aligned}
$$

\section{Step II (Figure 5)}

The values of the partial reliability blocks are:

$$
P_{p 6}(t)=P_{A 6}(t)+P_{P 1}(t), P_{P 7}(t)=P_{p 4}(t)+P_{p 1}(t) .
$$

Table 3. Values of transmission reliability functions of spinning box assembly depending on the value of its reliability.

\begin{tabular}{ccc}
\hline Reliability values $P_{i}(t)$ & Portable spinning subsystem reliability function $G_{P}(t)_{B P}$ \\
\hline 1.0 & 16 \\
0.9 & 8.078 \\
0.8 & 3.775 \\
0.7 & Shaded areas represent the & 1.6 \\
0.6 & ultimate limit of satisfactory & 0.5972 \\
0.5 & reliability in the analysis & 0.1875 \\
0.4 & & 0.0458 \\
0.2 & & 0.0006 \\
0 & & 0 \\
\hline
\end{tabular}

Note: The shaded areas included values because values below this limit are not taken into account (they include areas in which the assembly needs to be repaired, which will be discussed more when determining the reliability values in cases of selected statistical distribution). 


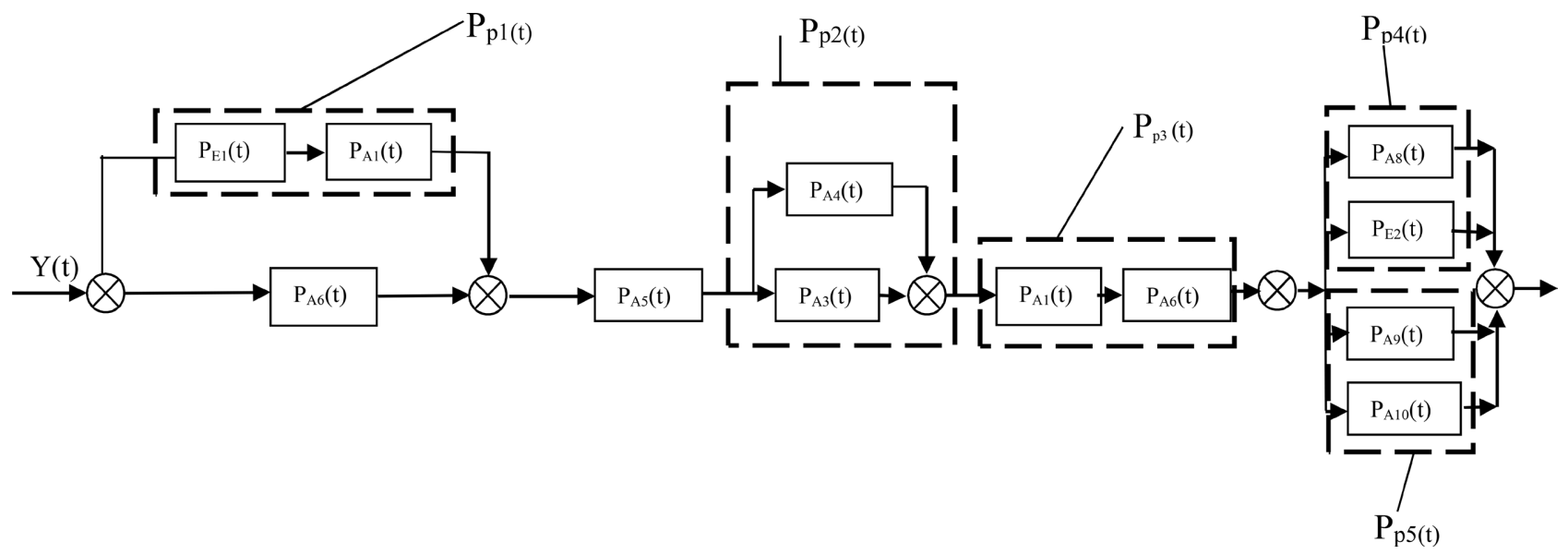

Figure 4. Initial model block diagram of the transmission reliability function of the spinning box assembly.

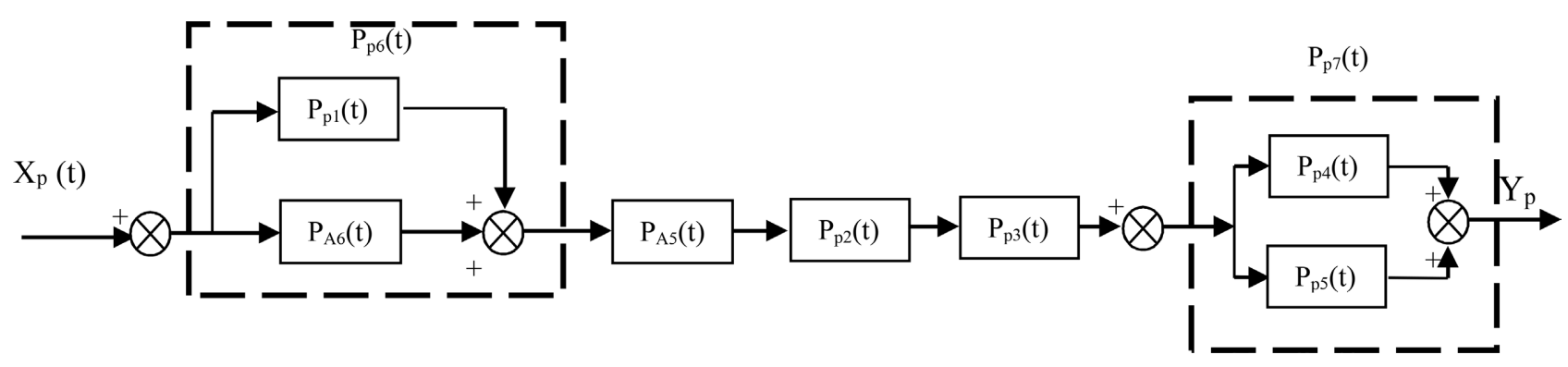

Figure 5. Block diagram reduction in step II for spinning box assembly.

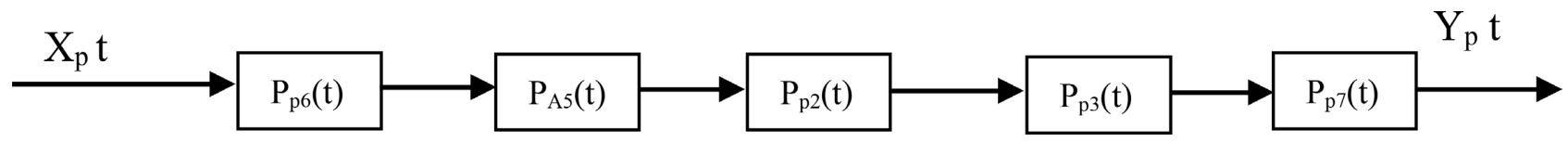

Figure 6. Block diagram reduction in step III for spinning box assembly.

\section{Step III (Figure 6)}

The values of the partial reliability blocks are:

$G_{B P}=P_{P 6}(t) \cdot P_{A 1}(t) \cdot P_{P 2}(t) \cdot P_{P 3}(t) \cdot P_{P 7}(t)$.

The final equation of the reliability value based on the partial reliability values for the box spinning assembly is:

$$
\begin{aligned}
G_{B P}(t)= & \frac{Y_{P}(t)}{X_{P}(t)}=\left(P_{A 6}(t)+P_{P 1}(t)\right) \cdot P_{A 5}(t) \cdot\left(P_{A 4}(t)+P_{A 3}(t)\right) \\
& \cdot P_{A 1}(t) \cdot P_{A 2}(t) \cdot\left(P_{P 4}(t)+P_{P 5}(t)\right) \\
= & \left(P_{A 6}(t)+P_{E 1}(t) \cdot P_{A 7}(t)\right) \cdot P_{A 5}(t) \cdot\left(P_{A 4}(t)+P_{A 3}(t)\right) \\
& \cdot P_{A 1}(t) \cdot P_{A 2}(t) \cdot\left(P_{A 8}(t)+P_{E 2}(t)+P_{A 9}(t)+P_{A 10}(t)\right) \\
= & P_{A 1}(t) \cdot P_{A 2}(t) \cdot P_{A 5}(t) \cdot\left\{\left(P_{A 6}(t)+P_{E 1}(t) \cdot P_{A 7}(t)\right)\right\}\left(P_{A 4}(t)+P_{A 3}(t)\right) \\
& \cdot\left(P_{A 8}(t)+P_{E 2}(t)+P_{A 9}(t)+P_{A 10}(t)\right) \\
G_{B P}(t)_{p}= & P_{A 1}(t) \cdot P_{A 2}(t) \cdot P_{A 5}(t) \cdot\left(P_{A 4}(t)+P_{A 3}(t)\right) \\
& \cdot\left\{\left(P_{A 6}(t)+P_{E 1}(t) \cdot P_{A 7}(t)\right)\right\}\left(P_{A 8}(t)+P_{E 2}(t)+P_{A 9}(t)+P_{A 10}(t)\right)
\end{aligned}
$$




\subsection{Table of the Transmission Function of the Spinning Box Assembly with Approximation $G_{P}(T)_{B P}$}

Table 3 is formed on the basis of the final expressions of the transfer reliability functions depending on the time interval of operation of the circuits.

The value of the transmission function of the spinning box assembly reliability is shown in a table (Table 3 ). Based on the obtained values, a graphical representation of the dependence was performed $f\left(G_{P}(t)_{B P}, t\right)$ (Figure 7 ).

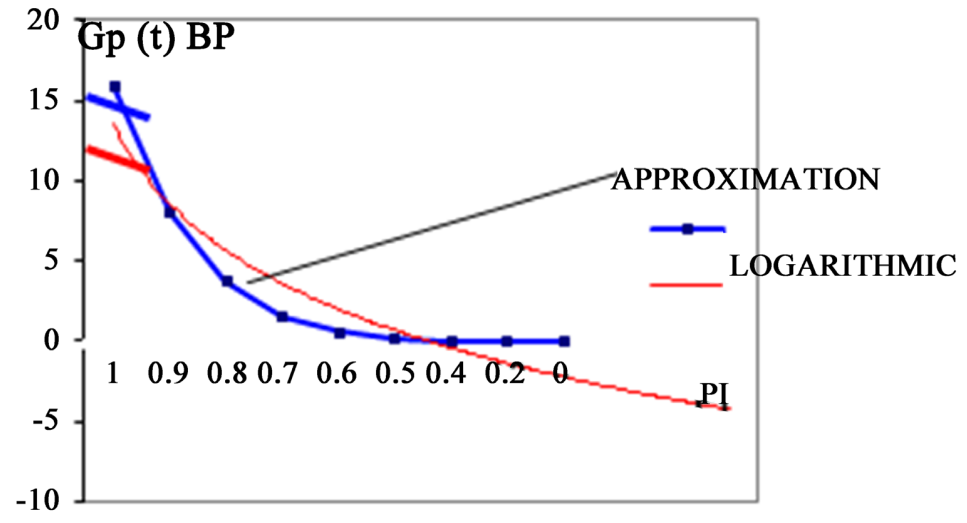

Figure 7. Graph of transfer function of spinning box with approximation $G_{P}(t)_{B P}$.

\section{Conclusion}

Guilty $f\left(G_{P}(t)_{B P}, t\right)$, corresponds to long normal curves according to their shape, so for that reason a long normal distribution will be taken for the selection of the statistical reliability distribution. According to this distribution, the reliability of each component of the analyzed assembly will be corrected. The optimal safety model includes the values of the operation of the components with the allowed risk, and it is clearly seen that the belt of absolutely safe operation of the analyzed circuit is located between the values of the displayed dependence curves $M_{\xi}(t)_{B P}=f(t)$. And also, analyses have been carried out to ensure the flawless operation of the $\mathrm{OE}$ machine and the consistency of product quality.

\section{Conflicts of Interest}

The authors declare no conflicts of interest regarding the publication of this paper.

\section{References}

[1] Blanchard, B. And Fabrysky, W. (1981) System Engineering and Analysis. Prentice Hall Inc., New Jersey.

[2] Brehmer, L. (1975) Technishe Diegnostik in der Flugzeuginstandhalting. Information der ziliven Luftfahrt, 11.

[3] Connor, P.M. (1981) Structured Analysis in Desing Technique. Sistems Analysis and Designa Foundation for the 1980, Norht_Holland, New York.

[4] Stefanovic, S. (2012) Determination of the Value of Selected Oscillation Frequency Measurement Point Analyzed Parts OE Spinning-On the Box Spinning. Internation- 
al Journal of Mechanical Engineering Research and Development, 2, 27-37.

[5] Stefanovic, S. (2006) The Influence of Mechanical Vibration on the Occurrence of Functional Safety Circuits in the Power Transmission System of Textile Machines. Ph.D. Thesis, University of Novi Sad, Novi Sad.

[6] Slobodan, S., et al. (2013) Research into the Causes of Inaccuracies of Components of Complex for Coil Winding with Finished Yern at OE. Journal of Process Management. New Technologies, 1, 16-26.

[7] Stefanovic, S. (2013) The Analysis of Functioning of Basic Components of OETechnical System. International Journal of Engineering, 11, 237-244.

[8] Korabayev, Sh.A., Mardonovich, M. , Lolashbayevich, M. and Xaydarovich, M. (2019) Determination of the Law of Motion of the Yarn in the Spin Intensifier. Engineering, 11, 300-306. https://doi.org/10.4236/eng.2019.115021 https://www.scirp.org/journal/paperinformation.aspx?paperid=92784

[9] Korabayev, Sh.A., Matismailov, S.L., Yuldashev, A.T. and Atanbayev D.D. (2020) Study of Fiber Movement Outside the Crater of Pnevmomechanical Spinning Machine. Solid State Technology, 63, 3460-3466.

http://www.solidstatetechnology.us/index.php/ISST/article/view/3473

[10] Dimitrijevic, N., Stefanovic, S., Mladenovic, S. and Vojislav, V. (2019) Use of Intelligent Systems for Electronic Stability of Motor Vehicles. Knowledge International Journal, 35, 961-966.

[11] Dimitrijevic, N., Stefanovic, S., Mladenovic, S. and Krstic, V. (2019) Use Of Gps Systems for Movement and Monitoring Vehicle in Road Traffic. Knowledge International Journal, 35, 955-960.

[12] Dimitrijevic, N., Stefanovic, S., Mladenovic, S. and Krstic, V. (2019) Use of Intelligent Systems on the Dynamic Stability of Cars. Knowledge International Journal, 35, 973-978. 